\title{
RESEARCH
}

Open Access

\section{Remodeling of the maternal gut microbiome during pregnancy is shaped by parity}

Alexander S. F. Berry ${ }^{1,2}$, Meghann K. Pierdon³, Ana M. Misic ${ }^{1}$, Megan C. Sullivan', Kevin O'Brien ${ }^{1}$, Ying Chen', Samuel J. Murray ${ }^{1}$, Lydia A. Ramharack', Robert N. Baldassano², Thomas D. Parsons ${ }^{3}$ and Daniel P. Beiting ${ }^{1 *}$ (D)

\begin{abstract}
Background: The maternal microbiome has emerged as an important factor in gestational health and outcome and is associated with risk of preterm birth and offspring morbidity. Epidemiological evidence also points to successive pregnancies - referred to as maternal parity — as a risk factor for preterm birth, infant mortality, and impaired neonatal growth. Despite the fact that both the maternal microbiome and parity are linked to maternalinfant health, the impact of parity on the microbiome remains largely unexplored, in part due to the challenges of studying parity in humans.
\end{abstract}

Results: Using synchronized pregnancies and dense longitudinal monitoring of the microbiome in pigs, we describe a microbiome trajectory during pregnancy and determine the extent to which parity modulates this trajectory. We show that the microbiome changes reproducibly during gestation and that this remodeling occurs more rapidly as parity increases. At the time of parturition, parity was linked to the relative abundance of several bacterial species, including Treponema bryantii, Lactobacillus amylovorus, and Lactobacillus reuteri. Strain tracking carried out in 18 maternal-offspring "quadrads" —each consisting of one mother sow and three piglets—linked maternal parity to altered levels of Akkermansia muciniphila, Prevotella stercorea, and Campylobacter coli in the infant gut 10 days after birth.

Conclusions: Collectively, these results identify parity as an important environmental factor that modulates the gut microbiome during pregnancy and highlight the utility of a swine model for investigating the microbiome in maternal-infant health. In addition, our data show that the impact of parity extends beyond the mother and is associated with alterations in the community of bacteria that colonize the offspring gut early in life. The bacterial species we identified as parity-associated in the mother and offspring have been shown to influence host metabolism in other systems, raising the possibility that such changes may influence host nutrient acquisition or utilization. These findings, taken together with our observation that even subtle differences in parity are associated with microbiome changes, underscore the importance of considering parity in the design and analysis of human microbiome studies during pregnancy and in infants.

Keywords: Pig, Pregnancy, Parity, Gut microbiome, Neonate microbiome, Early-life microbiota, $16 \mathrm{~S}$ rRNA sequencing, Shotgun metagenomics

\footnotetext{
* Correspondence: beiting@vet.upenn.edu

'Department of Pathobiology, School of Veterinary Medicine, University of Pennsylvania, Philadelphia, PA, USA

Full list of author information is available at the end of the article
}

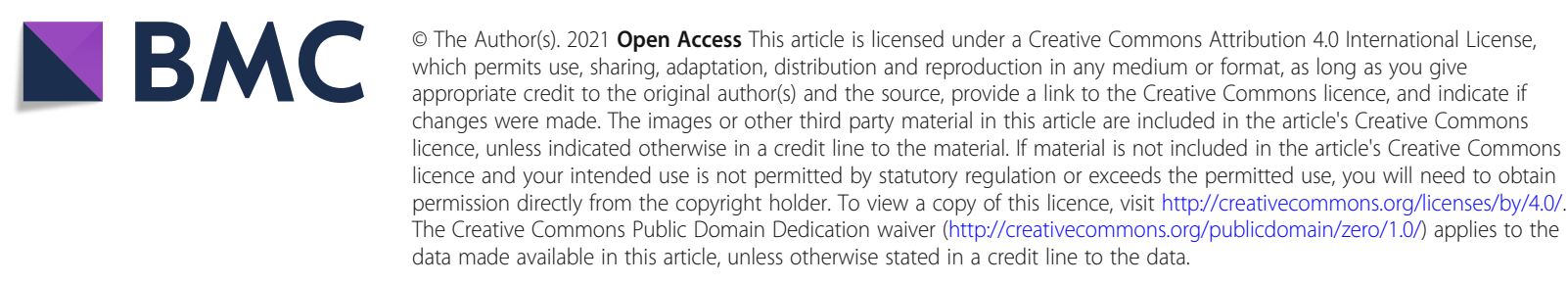




\section{Introduction}

The mammalian microbiome plays a key role in maternal and infant health, and recent studies have highlighted the value of the maternal microbiome for predicting the risk of preterm birth [1-3], the leading cause of neonatal death worldwide [4]. Although the exact mechanisms by which maternal microbes might influence pregnancy and offspring health have yet to be fully defined, studies in mice have begun to provide clues. For example, microbial metabolites produced in the maternal gut can be detected in the placenta and fetal tissues, where they drive postnatal innate immune development [5]. Similarly, short-chain fatty acids produced by the maternal microbiome cross the placenta, where they signal through multiple host pathways to protect offspring from metabolic disease [6]. Microbes are also vertically transmitted to offspring during birth and in the perinatal period, and these early colonizers can have long-term effects on child development [7-9]. Despite the growing recognition that the maternal microbiome influences infant health, we currently have a remarkably poor understanding of the clinical and environmental factors that can impact the microbiome during pregnancy.

Maternal parity-the number of previous pregnancies-is associated with increased risk of preterm birth in humans $[10,11]$, but studies of parity and health in humans are often confounded by numerous socioeconomic and psychosocial factors [12]. Since both the maternal gut microbiome and parity have been identified as key determinants of gestational health, it is important to understand whether parity influences the microbiome during pregnancy. Previous studies in dairy cows have shown that animals pregnant for the first time (nulliparous) have different uterine and rumen microbiome compositions than do animals with only a single prior pregnancy (primiparous) or two or more previous pregnancies (multiparous) [13, 14]. However, it is as yet unclear if parity impacts either the maternal gut microbiome during pregnancy or the microbiome of the developing offspring.

Elucidating the relationship between microbiome composition and pregnancy in human subject research is challenging. Studies are often characterized by small sample sizes [15], cross-sectional or sparse longitudinal sampling [16], and present challenges in controlling for confounding factors. Interpersonal variation in diet has a particularly large impact on gut microbiota composition in human studies [17], and differences in maternal diet during pregnancy have been shown to influence the infant gut microbiome [18]. Properly controlling for diet often involves either retrospective studies paired with self-reported food intake surveys [19], highly controlled feeding studies $[19,20]$, or a focus on geographically distinct populations that differ in dietary practices [21, 22], all of which are made more challenging in pregnancy. In addition, studies of pregnancy and the microbiome in humans rarely address parity due to limited numbers of pregnancies in most countries. Large animal models offer an appealing alternative to human studies for examining parity. Pigs are commonly used as biomedical models of humans due to similarities in anatomy and physiology and have provided valuable insight into functions of the human gut microbiome [23-25]. Here, we describe high-resolution microbiome profiling during synchronized pregnancy in sows, carried out in a highly controlled environment, to determine if the microbiome changes during pregnancy, and whether parity plays a role in this process.

16S rRNA marker gene sequencing and shotgun metagenomics were used to assess the association between pregnancy and the gut microbiome in a population of mother sows with parity ranging from zero to seven, where diet and environment are meticulously controlled. Maternal fecal samples collected weekly throughout the 114-day gestation, together with samples from piglets born to these mothers, allowed direct comparisons to be made between maternal and infant microbiomes. We observed that (1) the maternal gut microbiome changes predictably during pregnancy; (2) this remodeling occurs more rapidly in high-parity animals, compared to their low parity counterparts; (3) parity is associated with gut microbiome composition at parturition; and (4) the composition of the early infant gut microbiome is influenced by parity. Taken together, our results highlight the importance of considering parity in maternal gut microbiome studies and suggest that pregnancy history can shape both the maternal microbiome as well as early colonization events in offspring, albeit likely by different mechanisms.

\section{Results \\ Predictable changes in microbiome composition throughout pregnancy in the pig}

Previous studies examining the microbiome in pigs have focused either on broad stages of gestation [26], or on post-natal growth and feed efficiency [27-30]. In order to understand the extent to which the maternal gut microbiome is affected during pregnancy in pigs, stool samples were collected weekly from mother sows beginning at gestational day 34, when pregnancy was first confirmed by ultrasound, and throughout the full 114day gestation. Throughout, we will refer to samples collected on or before day 72 as early pregnancy, and samples collected prior to day 72 as late pregnancy as this is halfway through the sampling period and correlates with the first major shift in microbiome composition. The resulting 390 stools samples (10-12 per sow) were 
subjected to microbiome profiling by targeted sequencing of the V4 region of the $16 \mathrm{~S}$ rRNA gene. To leverage the longitudinal aspect of this data, we trained a supervised regression model on all samples from $60 \%$ of the animals to calculate a microbiome maturity index during pregnancy [31, 32], then tested the model on the remaining animals. This approach is ideal for longitudinal data because it quantifies the relative rate of change in microbiome composition over time. Defining microbiota maturity in such a way has been used to provide a microbial measure of development and is a useful tool for classifying microbial states or trajectories that deviate from expectation [32]. Our model identified a significant correlation $\left(P<3.3 \mathrm{e}-13 ; R^{2}=0.27\right)$ between the actual versus predicted day of gestation (Fig. 1A). Despite a trend toward overestimating day of gestation early in pregnancy and underestimating later, our model accurately predicted which samples belonged to lateversus early-term pregnancies. Training the model on the first six and last six time points independently resulted in a stronger correlation from days 79 to 114 than from days 37 to 72 (SFig 1A,B), suggesting that more changes occur later in pregnancy. The families Porphyromonadaceae and Muribaculaceae were the most important taxa for predicting day of gestation (Fig. 1B). To identify transitions in microbial community structure during pregnancy, we applied a Dirichlet multinomial mixtures (DMM), which uses a probabilistic approach to carry out unsupervised clustering of taxonomic data into community types. This analysis showed that the gut microbiome of most sows occupies cluster 1 from the time that pregnancy is confirmed until day 65 of gestation, indicating a shared microbiota structure early in pregnancy (Fig. 1C). However, as animals moved through pregnancy, the gut microbiome underwent a shift marked by a departure from cluster 1 , evident by day 72 (Fig. 1C). Treponema and Clostridium sensu stricto were the most important taxa for clustering gut microbiota communities by DMM (Fig. 1D and E). Taken together, the maturity index and DMM analyses show that the gut microbiome is remodeled during pregnancy.

\section{Parity modulates the pregnancy-induced remodeling of the gut microbiome}

Although our maturity index and DMM analyses show a clear predictable change in the microbiome during pregnancy, there was still substantial unexplained variation, as evident from the maturity index $R^{2}=0.27$. We hypothesized that parity may contribute to some of this variation. To test this hypothesis, we grouped animals based on whether they had no prior pregnancies (zero parity, or nulliparous; 107 samples from 9 animals), 1-3 prior pregnancies (low parity; 150 samples from 13 animals), or 4-7 prior pregnancies (high parity; 133 samples from 12 animals). A non-parametric microbial interdependence test (NMIT) was used to summarize data across all timepoints into a single microbiome trajectory value of each individual sow. Principal coordinate analysis (PCoA) (Fig. 2A; SFig 2) and NMDS (Fig. 2B) of these data showed that NMIT distances were significantly different between parity groups (Fig. 2C). The greatest difference in microbiome trajectory occurred between zero parity (nulliparous) and low parity sows, suggesting that having even one prior pregnancy was sufficient to impact microbiome trajectory during future pregnancies. The difference in microbiome trajectories between low and high parity sows was also significant (Adj. $P<0.05$ ), albeit less substantial than the difference between nulliparous and low parity animals.

To determine how the microbiome trajectory differs between parity groups, alpha and beta diversity metrics were compared across parity groups. Shannon alpha diversity increased throughout pregnancy across all parity groups and the rate of change did not depend on parity (SFig 3). Bray-Curtis beta diversity between each sample and the day 37 sample from the same animal was calculated. Both Bray-Curtis (Fig. 2D) and weighted UniFrac (SFig 4A) showed that nulliparous animals, compared to their low and high parity counterparts, exhibit a more subtle but incremental change from day 37 as pregnancy progresses. In contrast, high parity animals underwent a more abrupt change from day 37 early in pregnancy with little or no further change evident throughout. Since comparing each time point with a single reference likely fails to capture the magnitude of changes that occur between time points, we could not rule out that high parity sows might undergo substantial changes from week to week throughout pregnancy while remaining consistently different from day 37 . To reconcile these interpretations, beta diversity was calculated between each sample and the previous sample from the same individual using Bray-Curtis (Fig. 2E) and weighted UniFrac (SFig 4B). This analysis confirmed that the rate of change of gut microbiota composition in zero and low parity animals increases steadily throughout pregnancy, while high parity animals actually show a decrease over time, suggesting that as animals have more pregnancies, remodeling of the gut microbiome occurs more rapidly.

Since the largest differences in microbiome trajectory occur between zero and high parity animals, we further investigated the differences between these two groups over time by repeating the DMM modeling, but with animals grouped by parity. While most animals occupy the cluster 1 community type at day 37, regardless of whether they are high (Fig. 2F) or zero parity (Fig. 2G), high parity sows quickly move out of cluster 1 to occupy clusters $2,3,4$, and 5 throughout most of the pregnancy, 


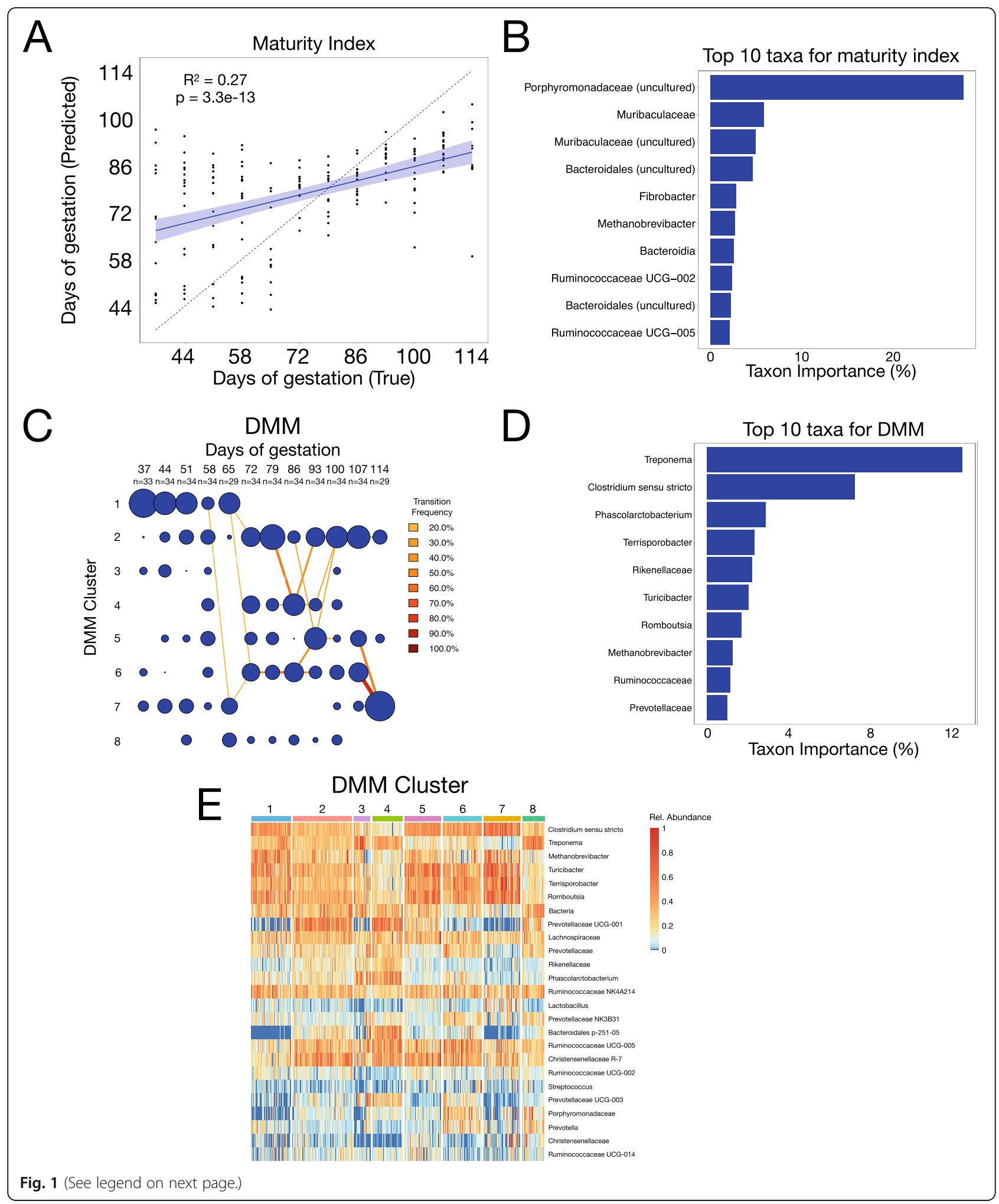


(See figure on previous page.)

Fig. 1 Gut microbiota compositional changes during gestation follow several predictable trends. 165 sequencing of fecal samples from 34 mother sows sampled longitudinally during the 114-day gestation reveals several trends in gut microbiota changes. A A maturity index trained on $60 \%$ of the animals and tested on the remaining $40 \%$ shows that the amount of time of gestation can be predicted $(P=3.3 \mathrm{e}-13)$ with some accuracy $\left(R^{2}=0.27\right)$ using gut microbiota composition data. B The 10 taxa that contributed the most to the accuracy of the maturity index are shown in order of importance. C Dirichlet multinomial mixtures (DMM) bin samples into one of 8 clusters, each defined by a unique gut microbiota composition. Days of gestation are on the X-axis, and clusters are labeled on the Y-axis. The total number of samples at each time point is also labeled on the X-axis. The size of the blue circle is proportional to the number of samples contained in each cluster. Transitions made by a substantial percentage of individuals (transition frequency $>20 \%$ ) are indicated by lines connecting blue clusters. D The 10 taxa that contributed the most to the accuracy of the DMM are shown in order of importance. $\mathbf{E}$ Heatmap shows the relative abundance of taxa (normalized across each taxa) for each sample. Samples are grouped by DMM cluster to allow for visualization of the taxa that are enriched or reduced on average in each DMM cluster

with most of these animals occupying cluster 2 at fullterm. Interestingly, nulliparous sows also leave cluster 1 early in pregnancy but occupy clusters 6,7 , and 8 instead (most in cluster 7 at full-term)-three clusters rarely, if ever, occupied by high parity sows at any point during pregnancy (Fig. 2F, G). A spline-fitting model was applied to the top 5 most important taxa from the DMM and top 5 from the maturity index. This analysis showed that the relative abundance of all ten taxa changed throughout pregnancy and that the relative abundance of Phascolarctobacterium and Rikenellaceae was significantly associated with parity (SFig 5). Taken together, the data show that parity influences the gut community types that develop during pregnancy.

\section{Parity is associated with an altered microbial environment during the perinatal period}

Our DMM analysis suggested that by the end of gestation, high parity animals had a community type that was distinct from that of zero parity animals. To better understand how parity influenced the microbial environment as animals approached parturition, we carried out shotgun metagenomic sequencing on a subset of the animals profiled above, along with their offspring. In total, 18 mother-offspring "quadrads"-each comprising a mother sow and three of her piglets-were examined, including stool from 7 nulliparous sows and 11 high parity sows at day 37 and at the end of gestation (day 114), along with rectal swabs collected from 54 piglets at day 10 of life. Bray-Curtis beta diversity was calculated among high parity sows and between high and zero parity sows at both timepoints. At day 37 , parity is only slightly correlated with beta diversity $\left(P=0.050 ; R^{2}=\right.$ $0.11)$ (Fig. 3A). Conversely, parity is strongly correlated with beta diversity by day $114\left(P=9 \mathrm{e}-4 ; R^{2}=0.21\right)$ (Fig. $3 \mathrm{~B})$. To identify the species driving these community composition differences, differentially abundant taxa were calculated using linear discriminant analysis (LEfSe) at both timepoints. Consistent with the beta diversity analysis, only two low-abundance taxa were differentially abundant between zero and high parity animals at day 37: Selenomonas bovis and Prevotella copri, both of which were more abundant among zero parity animals compared to those of high parity (Fig. 3C). At day 114, six taxa were differentially abundant between zero and high parity including Methanobrevibacter (unclassified), Porcine type C oncovirus, Peptostreptococcaceae (unclassified), Treponema bryantii, Lactobacillus amylovorus, and Lactobacillus reuteri (Fig. 3D). The differences in beta diversity and differentially abundant taxa at each timepoint suggest that the impact of parity on microbiome composition is modest early in pregnancy and that parity-associated taxa emerge later as animals near parturition.

\section{Maternal gut microbiome composition does not predict early infant gut colonization}

Our observation that the sow gut microbiome was influenced by parity, together with recent evidence suggesting that maternal gut microbes are likely candidates for vertical transmission to offspring [33, 34], prompted us to characterize the relationship between maternal and offspring gut microbes. As expected, Bray-Curtis beta diversity analysis of metagenomic data from piglets born to each sow $(n=54)$ showed that maternal gut microbiomes were markedly distinct from those of the piglet gut ( $P=1 \mathrm{e}-5, R^{2}=0.27$ ), such that the gut microbiome composition of a sow is more similar to that of other sows than to that of her infant offspring (SFig 6A). Surprisingly, there was no relationship between the relative abundance of the dominant species observed in the maternal sow gut and those in their piglets. Many microbes present in the piglet gut were completely absent from the maternal gut and vice versa (SFig 6B), and a linear regression comparing the relative abundance of the eight most abundant bacterial taxa in the maternal gut at day 114 to the same taxa in the offspring gut showed no correlation (Adj. $P>0.3$ ) (SFig 7). To explore this in more detail, we focused on Escherichia coli, which was present at the high relative abundance in both sows and piglet, and performed strain tracking using StrainPhlAn [35] to determine whether the mother and offspring harbored the same strain. After removing samples for which multiple $E$. coli strains were detected and samples for which 


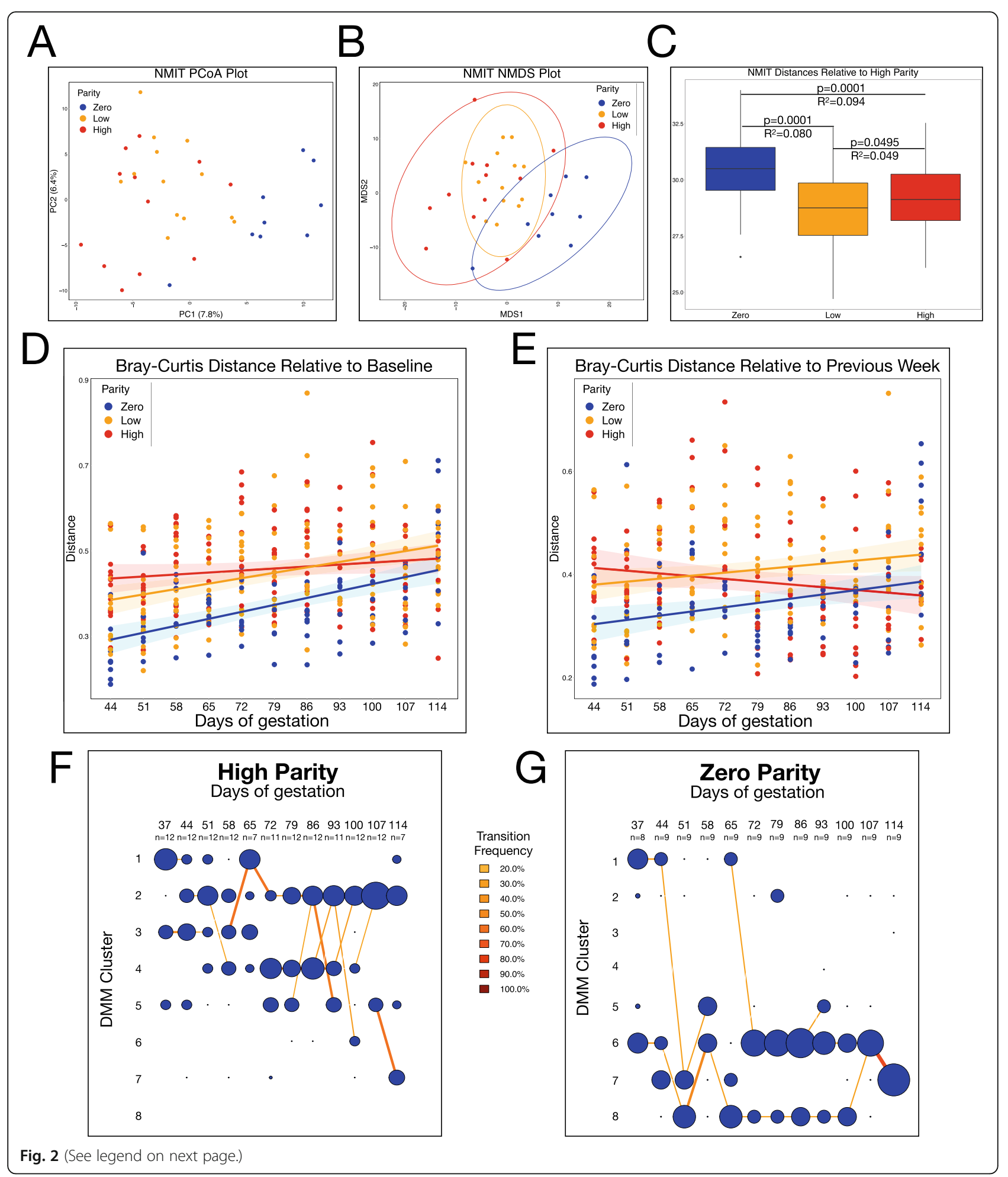


(See figure on previous page.)

Fig. 2 Parity affects the gut microbiota trajectory during gestation. Clustering each of the 34 individuals by parity (zero = no previous pregnancies, low $=1-3$ previous pregnancies, high $=4-7$ previous pregnancies) reveals that parity explains a significant amount of the variation between gut microbiota trajectories during gestation. A Non-parametric microbial interdependence test (NMIT), which calculates correlations between each pair of taxa for each individual over time, was performed for each individual. A principal coordinate analysis (PCoA) plot of the NMIT data shows how the gut microbiota trajectory differs between animals of different parities across the first two axes. B Non-metric multidimensional scaling (NMDS) plot of the NMIT data depicts the differences between the gut microbiota trajectories, with $95 \%$ confidence intervals displayed as ellipses around each parity bin. C Boxplots depict the differences in NMIT between each individual and each high parity individual. Boxplots show the median and the first and third quartiles, with whiskers that extend to outliers up to 1.5 times the interquartile range. The adjusted $P$ values and $R^{2}$ are shown for each comparison. $\mathbf{D}$ Bray-Curtis beta diversity was calculated between each sample and the day 37 sample from the same individual. E Bray-Curtis beta diversity was calculated between each sample and the previous week's sample from the same individual. $\mathbf{F}$ Dirichlet multinomial mixtures (DMM) showing only samples collected from high parity animals. G DMM showing only samples collected from zero parity animals
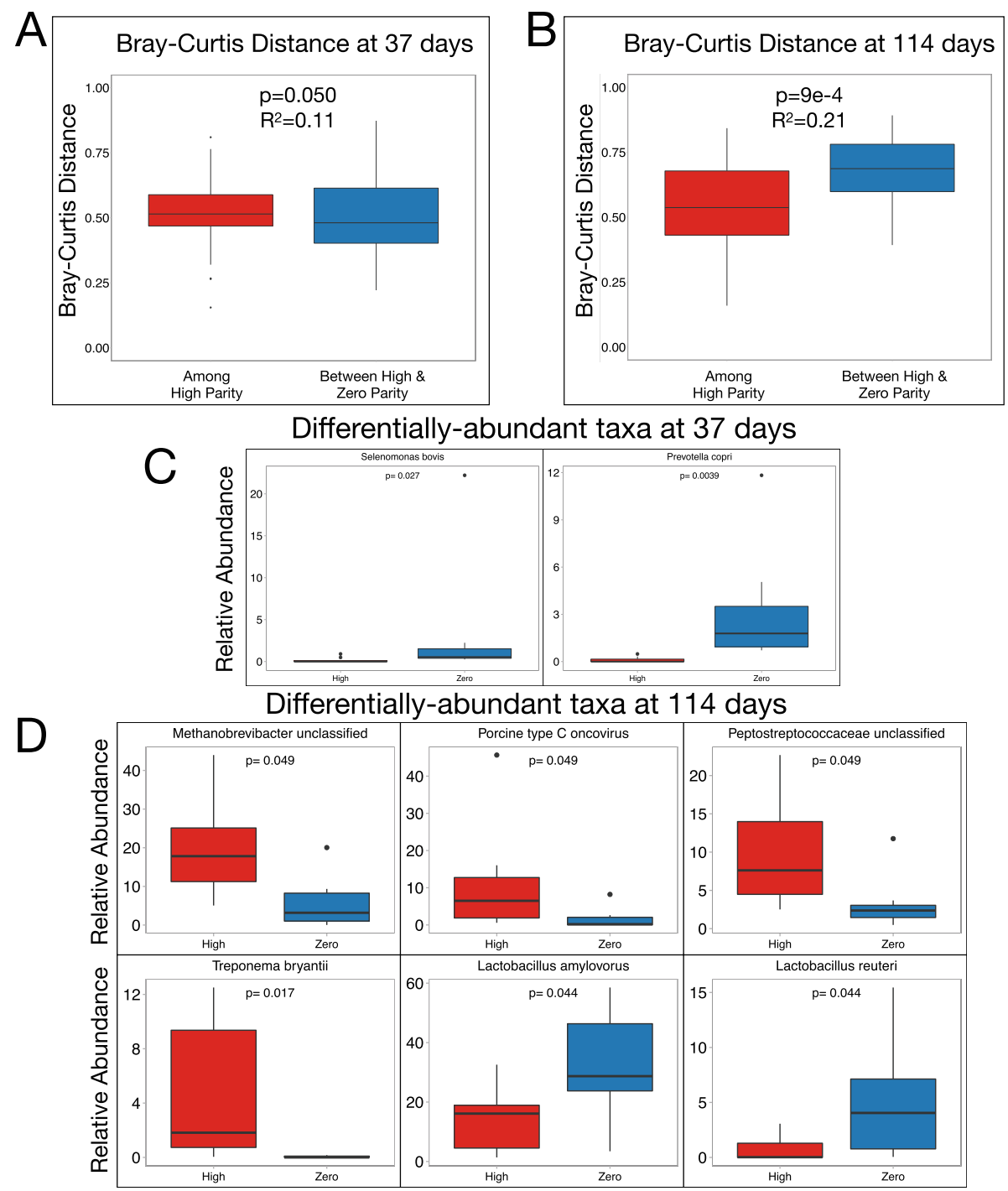

Fig. 3 Parity is associated with significant differences in the relative abundance of key taxa at the end of gestation. Fecal samples from 18 mother sows (7 of parity zero and 11 of parities 3-7) were collected at days 37 and 114 of gestation, and shotgun metagenomic sequencing was performed. Bray-Curtis beta diversity was calculated between each sample and each high parity sample at $\mathbf{A}$ the beginning of gestation (day 37 of gestation) and $\mathbf{B}$ just prior to delivery (day 114 of gestation). All microbes with average relative abundance $>1 \%$ across all 36 samples (Adj. $P<$ 0.05) that were differentially abundant between zero and high parity animals at $\mathbf{C}$ day 37 and $\mathbf{D}$ day 114 are shown. Boxplots show the median and the first and third quartiles, with whiskers that extend to outliers up to 1.5 times the interquartile range. $P$ values (adjusted for multiple testing in $\mathbf{C}$ and $\mathbf{D}$ and $R^{2}$ are shown where appropriate 


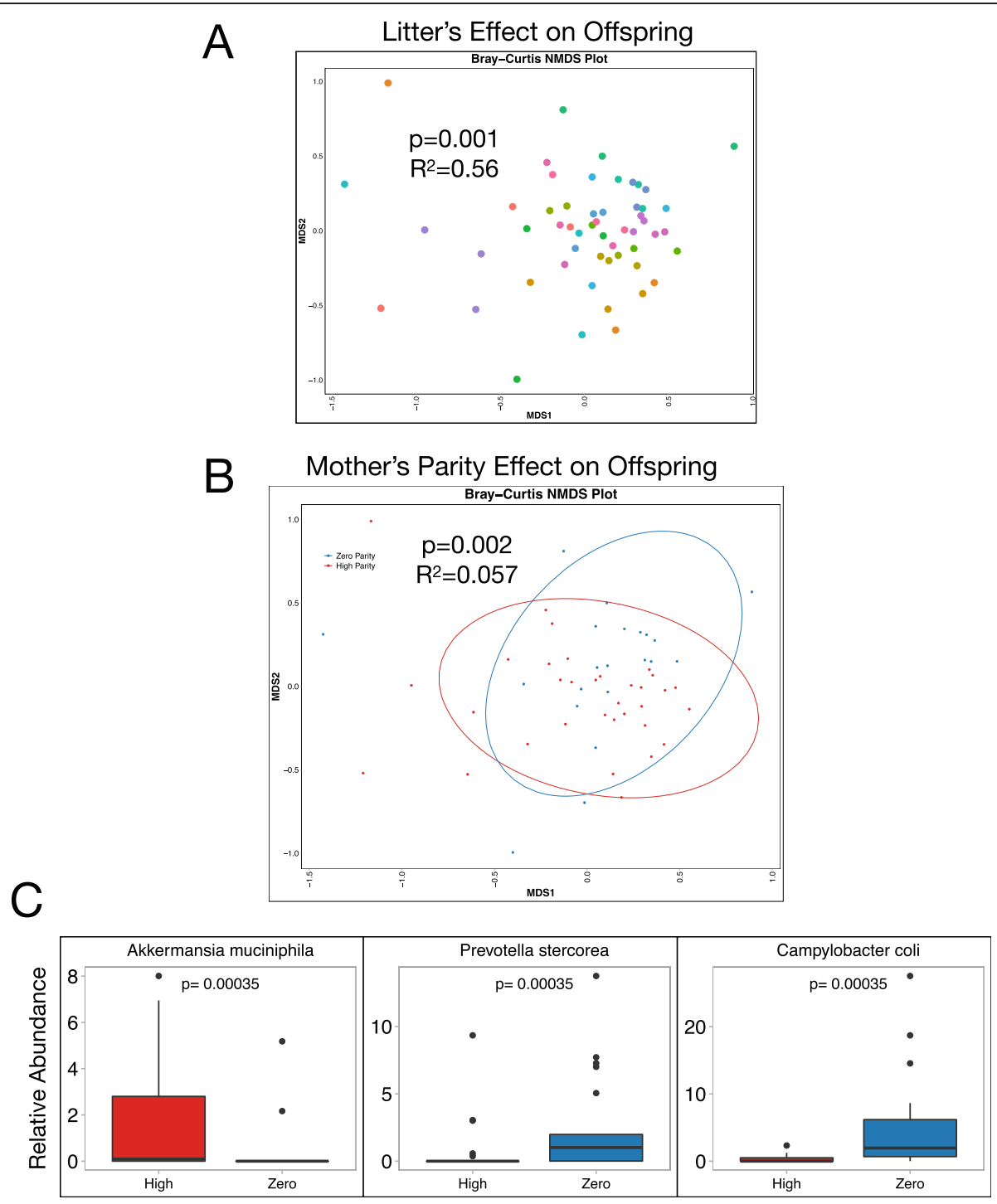

Fig. 4 Maternal parity is associated with significant differences in offspring gut microbiome composition. Fecal swabs from 3 offspring of each of the 18 mother sows were collected 10 days after delivery and shotgun metagenomic sequencing was performed. A An NMDS plot shows the differences in gut microbiome composition across all 54 piglets, as determined by Bray-Curtis distance. Piglets born to the same mother (representing a unique "litter") are represented by the same color. Litter is significantly associated with gut microbiome composition $(P=0.001)$ and explains most of the variation in gut microbiome composition $\left(R^{2}=0.56\right)$. B The NMDS plot comparing the Bray-Curtis dissimilarity between piglet gut microbiome samples is colored by the parity of the piglet's mother. Maternal parity is significantly associated with gut microbiome composition ( $P=0.002)$ and explains $5.7 \%$ of the variation in gut microbiome composition $\left(R^{2}=0.057\right)$. C All microbes with average relative abundance $>1 \%$ across all 54 piglet fecal samples (Adj. $P<0.05$ ) that were differentially abundant between those born to zero and high parity mothers are shown. Boxplots show the median and the first and third quartiles, with whiskers that extend to outliers up to 1.5 times the interquartile range. Adjusted $P$ values are shown for each differentially abundant taxa

no $E$. coli was detected, a neighbor-joining phylogeny confirmed that $E$. coli strains observed in siblings are more closely related than $E$. coli observed in non-siblings, but also revealed that a mother and her offspring harbor distinct strains of $E$. coli (SFig 8). Taken together, these data suggest that the most abundant early colonizers of the piglet gut are unlikely to have originated from the maternal gut.

\section{Maternal parity is associated with altered microbiome composition in offspring}

Our analysis of $E$. coli strains in metagenomic data from piglets suggested similarities in gut microbiomes of animals from the same litter. Indeed, Bray-Curtis beta diversity comparing all 54 piglets showed that litter-or mother to which a piglet is born-is significantly associated with gut microbiome composition 10 days after birth and explains more than half of the variation 
between piglet gut microbiomes $\left(P=0.001, R^{2}=0.56\right)$ (Fig. 4A). These data indicate that, although few taxa are shared between mother and offspring in our study, maternal factors are still a major driver of the offspring microbiome. We therefore hypothesized that maternal parity could influence the offspring microbiome in ways that are independent of direct vertical transmission of taxa. Consistent with this notion, maternal parity was also significantly associated with piglet gut microbiome composition $\left(P=0.002, R^{2}=0.057\right)$ (Fig. $\left.4 \mathrm{~B}\right)$. The differences between offspring born to nulliparous versus high parity mothers were driven by three bacterial taxa: Akkermansia muciniphila, Prevotella stercorea, and Campylobacter coli (Fig. 4C). Notably, litter size, litter weight at birth, and sow interpregnancy interval were not different between parity groups, suggesting that these factors do not contribute to the differences observed in gut microbiome composition between animals born to nulliparous versus high parity sows (SFig 9). Moreover, none of the bacterial species identified in this analysis were found to be differentially abundant between nulliparous and high parity sows, arguing that parity impacts both maternal and offspring gut microbial communities, but that the mechanisms by which this occurs likely differs.

\section{Discussion}

Longitudinal analysis of the gut microbiome in 34 mother sows revealed a shift in the community types present during gestation. A limited number of longitudinal studies have been conducted during pregnancy in humans, with one study reporting a dramatic shift in community composition from first to third trimester [16], while another showed remarkably stable community structure in the gut, vagina, and oral cavity throughout gestation [2]. These conflicting results highlight that additional data from well-controlled animal studies, as well as from diverse human populations-such as the ongoing Multi-Omic Microbiome Study: Pregnancy Initiative (MOMS-PI) [3] - are needed to generate a more complete picture of host-microbiome interactions during pregnancy. Recent efforts to consider statistical methods for analysis of longitudinal microbiome data and normalization for cross-study comparisons [36, 37], together database efforts that enable integration of large volumes microbiome data [38], all constitute important developments that will enhance our ability to integrate and mine microbiome data from different types of maternal-infant microbiome studies.

Our results show that the maternal gut microbiome undergoes consistent changes during pregnancy. Although stool samples were first collected from sows when they were confirmed pregnant at $\sim 37$ days postmating, rather than prior to or at the start of pregnancy, our data suggest that the gut microbiome remains similar throughout the first two months of pregnancy (Fig. 1C, SFig 1A, Fig. 3A). Importantly, data from swine fetal development studies point to major shifts in fetal growth and metabolism that occur within our sampling period. For example, the results of our DMM analysis showed a marked shift in microbiome composition starting around day 72 (Fig. 1C), which is coincident with remarkable metabolic shifts in both the sow and the developing fetus, including a dramatic acceleration of fetal weight gain, as well as accumulation of fetal protein and sow mammary gland protein content [39]. Whether the sow microbiome, and pregnancy- or parity-associated changes in the microbiome, play any role in these processes merits further investigation.

Our data show that parity modulates gut microbiome maturation during gestation in pigs. One confounding factor in the study of parity in both our experiments as well as in human studies is age, since humans and animals of higher parity are also usually older. In addition, pigs are highly social and establish a social hierarchy based on age. Consequently, nulliparous pigs are not only the youngest animals but are also at the bottom of the social rank and would normally be subject to bullying by older animals of higher parity. To mitigate unwanted effects of social hierarchy and ensure maximum animal welfare, nulliparous and multiparous sows were separated by a fence during gestation (see the "Methods" section). However, several lines of evidence argue against age being a major contributor to the differences observed in our parity analysis. First, we showed that gut microbiome composition at day 37 of pregnancy is similar between nulliparous (younger) and multiparous (older) animals, despite the fact that these groups have been separated for approximately five weeks (Fig. 3A, C). Furthermore, DMM analysis also showed that most animals, regardless of parity and age, occupy the same cluster at day 37 . Second, approximately 5 months of age typically separate sows that differ by a single parity, yet the NMIT analysis revealed that the largest change in microbiome trajectory occurs between nulliparous and single parity sows (SFig 2), rather than between the very youngest and oldest animals. Lastly, the impact of parity on the microbiome is evident even when the nulliparous (youngest) animals are excluded-high and low parity animals are not separated by the fence, yet they still differ in microbiome maturation during pregnancy (Fig. 2, SFig 1B).

Despite the fact that anaerobic bacteria identified in our piglet gut samples, including Bacteroides species and the sulfate reducer Disulfovibrio piger [40], are common members of the gut microbiome, our paired metagenomic analysis of maternal-offspring "quadrads" suggested that the piglet neonatal gut microbiome does not 
originate from the maternal gut (SFigs 3, 4, 5). However, we examined piglets only at a single time point, and the reference-based metagenomic analysis we used may lack the sensitivity to detect low abundance organisms; thus, we cannot rule out the possibility that some early colonizers of the infant gut were inherited from the maternal gut. Nevertheless, the apparent lack of shared strains or species between the maternal gut microbiome at parturition and the piglet gut at day 10 of life suggests that early gut colonizers are acquired, at least in part, from other maternal or environmental sources. Interestingly, numerous Lactobacillus species as well as Clostridium clostridioforme were detected in the piglet gut, but were absent from the sow gut, and have been observed in human vaginal microbiome studies [41, 42]. Subdoligranulum, a genus found in nearly all our piglets, has been identified in breast milk microbiomes [43]. In humans, breastfeeding is a source of early infant gut microbes [44] and is a significant predictor of early childhood gut microbiota [45] and immunity [46]. Parity has been shown to affect the lipid and protein content in human breast milk [47], as well as the microbiota composition of cow colostrum [48]. Taken together, these data suggest that the vaginal mucosa and breast milk should be explored for their potential as early sources of microbial colonization of the piglet gut, and our piglet data raise the possibility that one or both sites may be influenced by parity. In addition, a multi-niche analysis of the farrowing environment may yield insight into the origin of specific bacterial strains found in the piglet gut but not the maternal gut.

We identified several parity-associated bacterial species in the maternal gut at the end of gestation, as well as in the piglet gut. Among these were Treponema, the top taxon in our DMM model of the gut microbiome during gestational (Fig. 1D), and specifically the species $T$. bryantii which was identified as enriched in high parity pigs by metagenomic sequencing (Fig. 3D). Although little is known about this spirochete, T. bryantii was recently linked to increased feed efficiency in sows [49]. Lactobacillus levels ere peaked in DMM cluster 7 (Fig. $1 \mathrm{E})$, the cluster occupied by most nulliparous animals at the end of gestation (Fig. 2G). Two species, L. reuteri and L. amylovorus, were enriched among nulliparous animals (Fig. 3D), and both are common probiotic strains associated with weight and fat gain, and enhanced immune function in sows and piglets [50-53]. We also found that piglets born to high parity sows had increased relative abundance of Akkermansia muciniphila (Fig. 4 C), a species recently identified in human breast milk and breast tissue [54] and which has been widely linked to reduced risk of obesity and metabolic disease in humans $[55,56]$ and more recently to reduced adiposity in pigs [57]. Collectively, these data support a model whereby parity-associated changes in the microbiome have the potential to alter maternal or infant metabolism.

Given the causal role for the microbiome in a wide range of human diseases, our data suggest that the microbiome merits further exploration as a possible contributing factor to parity-associated outcomes in maternal and infant health. For example, it is well established that parity reduces the risk for estrogen receptor- and progesterone receptor-positive breast cancers [58-60] and ovarian cancer [61], but increases the risk of dental disease [12] and dementia [62]. Although the mechanisms underlying these parity-associated effects remain unclear, studies point to long-term alterations in hormones and systemic inflammatory mediators as possible contributors [63-65]. Just as we observed an impact of parity on the offspring gut microbiome, so too are there well-documented effects of parity on infant morbidity and mortality. Offspring born to nulliparous mothers have reduced birth weight and higher mortality rates [60] and are at increased risk of childhood obesity and metabolic disease [66]. Whether and how these parityassociated phenotypes and diseases are linked to the microbiome remains an important and unresolved question. Future studies exploring this topic will be valuable not only in improving agriculture, but also for advancing microbiome-based diagnostics and therapies to improve maternal-infant health in humans.

\section{Conclusions}

Pregnancy history affects both the maternal gut microbiome during gestation and the infant gut microbiome postpartum. Sources other than the maternal gut are potential contributors to the early piglet microbiome. Parity influences the relative abundance of key bacterial species associated with obesity and altered metabolism, including Akkermansia muciniphila, Treponema bryantii, and several Lactobacillus species.

\section{Methods}

\section{Animal husbandry and sample collection}

Fecal samples for our Pig Pregnancy and Parity (P3) Microbiome study were acquired from animals housed at the Penn Vet Swine Teaching and Research Center. The facility was environmentally controlled and maintained high standards of hygiene. Study animals were selected from one of two adjacent pens of gestating sows in the barn's gestation area. The first pen housed a group of 130 gestating parous sows (having previously birthed a litter) that were maintained in a single large dynamic group and fed by two electronic sow feeding (ESF) stations (Compident VII, Schauer Agrotronics, Prambachkirchen, Austria). The second pen housed a similar, but smaller group, of 65 nulliparous sows (not 
having previously birthed a litter) in a single dynamic group and were fed by a single ESF station. The smaller, younger animals were separated from the older sows to help mitigate the stress and other unwanted impact of conspecific aggression that arises in the establishment of a social hierarchy as older sows tend to bully the younger animals. The pens adjoined and created a near identical physical environment for the sows but were separated by a fence line that prevented direct nose to nose contact between groups. Both groups were subjected to similar management and husbandry practices although the larger group had access to straw bedding and an outdoor concrete loafing area. These pens provide animals with a space allowance of at least $2.0 \mathrm{~m}^{2} /$ head. Weaned, mated animals were added to the dynamic groups weekly at 8 days post-weaning. A similar number of near-term pregnant animals (>day 110 of gestation) were removed from the gestation pens and transferred to the farrowing and lactation area of the barn. Sows gave birth in individual pens $\left(4.1 \mathrm{~m}^{2}\right)$ equipped with a hinged farrowing crate. This crate initially provided protection to the newborn piglets but was opened at 10 to 14 days post-partum to provide the sow with additional mobility. Sows in the birthing pens only came into contact with their own piglets, and the newborn piglets only contacted one another and their mother for the duration of lactation. Piglets were weaned ranging from 28 to 35 days of age. Weaned sows were moved to the breeding area of the barn and housed in individual stalls while they were bred via post-cervical artificial insemination prior to returning to the gestation pens. The birthing pens were pressure washed with hot water and detergent to remove all organic matter and then disinfected prior to refilling with another near-term sow. The number of piglets per litter, interpregnancy interval, and litter birth weight were similar across all parity groups (SFig 9).

All sows were fed a similar standard corn-soy diet meeting or exceeding NRC [67] standards for gestating and lactating sows with a metabolizable energy of 3197.2 $\mathrm{kcal} / \mathrm{kg}$. The quantity of feed each sow received was based on both stage of gestation or lactation and body condition. Animals with less condition (skinnier) received larger quantities of feed than animals with more condition (fatter). Body condition of animals was scored at placement into the gestation pen and then reevaluated at $\sim 30$-day intervals. Standard production metrics were collected for each sow and their litter including sow age and parity (number of previous pregnancies resulting in a birth). Sows were confirmed pregnant at $\sim 37$ days post-mating via real-time ultrasonography. In order to determine how the gut microbiome changed throughout pregnancy, a fecal sample was collected from each pregnant sow at the time of pregnancy confirmation (37 days of gestation) and every 7 days until delivery ( $\sim 114$ days of gestation), for a total of 12 fecal samples per sow. To determine the effect of maternal parity and gut microbiome composition on the gut microbiome composition of offspring, fecal swabs were taken from piglets 10 days after birth. Fecal samples and swabs were stored at $-80^{\circ} \mathrm{C}$ until DNA extraction.

\section{S rRNA gene sequencing and processing}

$16 \mathrm{~S}$ rRNA gene sequencing was carried out on stool samples collected weekly from 34 sows throughout gestation. This group included 9 zero parity animals (no previous pregnancies), 13 low parity animals (1-3 previous pregnancies), and 12 high parity animals (4-7 previous pregnancies). Samples were obtained from at least 10 unique time points from each sow, with most sows represented by all 12 sampling times (weekly from day 37 to 114), for a total of 390 total fecal samples. DNA was extracted from fecal samples using Qiagen PowerSoil DNA extraction kit. 16S rRNA sequencing was performed as described previously [68]. Briefly, the V4 region of the 16S rRNA gene was amplified using PCR using Accuprime Pfx Supermix and custom primers for 30 cycles [69]. Quantification and clean-up of post-PCR products was carried out using PicoGreen reagent and AMPureXP beads, respectively. Pooled PCR libraries were quantified and sized using a Qubit 2.0 and Tapestation 4200, respectively. 250bp paired-end sequencing was performed using an Illumina MiSeq. The QIIME2 pipeline [70] was used to process and analyze $16 \mathrm{~S}$ sequencing data using qiime 2 version 2019.7.0. Samples were demultiplexed using q2-demux and denoised using Dada2 [71]. Sequences were aligned using maaft [72], and phylogenetic trees were reconstructed using fasttree [73]. Weighted UniFrac [74] and Bray-Curtis [75] beta diversity metrics were estimated using q2-core-metricsdiversity after samples were rarefied to 10000 reads per sample, and $p$ values were adjusted for multiple hypothesis testing using Benjamini-Hochberg (B-H) false discovery rate (FDR) corrections [76]. Taxonomy was assigned to sequences using q2-feature-classifier classifysklearn [77] against the Silva rRNA reference database $[78,79]$. Taxa were collapsed to the genus level, when possible. OTUs with less than $0.1 \%$ average relative abundance across samples, and those present in less than half of samples, were removed.

\section{Longitudinal data analysis}

The qiime2-longitudinal plugin was used to analyze weekly sampled microbiome data from each sow [80]. A maturity index was calculated using $60 \%$ of the samples as training data to compare the expected and predicted days of gestation using qiime longitudinal maturityindex $[31,32]$. To determine whether parity affects 
microbiome remodeling during pregnancy, the change in Bray-Curtis and weighted UniFrac beta diversity metrics over time were determined using qiime longitudinal first-distances and qiime longitudinal linear-mixedeffects. Non-parametric microbial interdependence tests (NMIT) were performed using qiime longitudinal nmit [81]. NMIT calculates correlations between each pair of taxa for each individual over time, allowing direct, quantitative comparisons of microbial interdependence between individuals as opposed to between timepoints within an individual. Dirichlet multinomial mixtures (DMM) was used to model the relationships between microbial communities as determined by $16 \mathrm{~S}$ sequencing using R code modified from Stewart et al. 2018 [82, 83], and DMM clusters (community types) were determined based on lowest LaPlace approximation. The proportion of samples occupying a DMM cluster at each timepoint was plotted to visualize changes in community types over time during pregnancy. SplinectomeR was used to determine whether the relative abundance of key bacterial taxa changed during the course of pregnancy and to determine whether the change in relative abundance was affected by parity [84].

\section{Shotgun metagenomic sequencing and analysis}

DNA extracted from fecal samples obtained from 18 of the study animals early (day 37) and late in gestation (day 114) were used for shotgun metagenomics. This subset included 7 zero parity sows and 11 high parity sows (parity $\geq 3)$ ( $n=36$ samples). DNA was also extracted from fecal swabs collected from 3 piglets from each of the 18 sows $(n=54)$. All DNA extractions were carried out using the Qiagen PowerSoil DNA extraction kit, and sequencing libraries $(n=90)$ were prepared following Illumina's Nextera XT protocol. Sequencing was performed on a NextSeq500 to generate $150 \mathrm{bp}$ singleend reads. Reads were trimmed using trimmomatic version 0.33 [85], and the quality was confirmed using FastQC. MetaPhlAn version 2.6.1 was used to determine the relative abundance of microbial taxa in each sample, collapsed to species [86]. The correlation between variables such as parity and microbiota composition was determined using PERMANOVA as implemented in the vegan package [87] in $R$ [88]. Differentially abundant taxa were determined using LDA effect size (LEfSe) [89], and $p$ values were adjusted for multiple hypothesis testing using B-H FDR corrections in R. Nonmetric multidimensional scaling (NMDS) plots comparing sow and piglet microbiome composition were generated using the vegan package in $\mathrm{R}$. Plots were visualized using ggplot2 [90], patchwork [91], and ggthemes [92]. Heatmap was generated using hclust2 (available at https:// github.com/SegataLab/hclust2). To determine whether species found in the piglet gut originated from the maternal gut, markers from Escherichia coli, a microbe found in high abundance across sow and piglet guts, were extracted using StrainPhlAn [35]. Samples for which multiple strains were suspected were removed from the analysis. A neighbor-joining phylogenetic tree was reconstructed with 1000 bootstrap replicates using MEGA7 [93] and visualized using FigTree version 1.4.4 (available at http://tree.bio.ed.ac.uk/software/figtree/).

\section{Supplementary Information}

The online version contains supplementary material available at https://doi. org/10.1186/s40168-021-01089-8.

Additional file 1: Supplemental Figure 1. Gut microbiota compositional changes more predictably after day 72 of pregnancy. A maturity index trained on $60 \%$ of the animals and tested on the remaining $40 \%$ from $A$ ) days 37 to 72 shows that the amount of time of gestation can be predicted $(P=8.9 \mathrm{e}-4)$ but with low accuracy $\left(R^{2}=0.12\right)$ and from $B$ ) days 79-114 shows that the amount of time of gestation can be predicted ( $P=8.5 \mathrm{e}-7)$ with higher accuracy $\left(R^{2}=0.25\right)$.

Additional file 2: Supplemental Figure 2. The most significant difference between gut microbiota trajectories lie between nulliparous and multiparous animals. A Principal Coordinate Analysis (PCoA) plot of the NMIT data shows how the gut microbiota trajectory differs between animals of different parities across the first two axes. Each point represents an individual's trajectory during gestation. Each point is a number which represents the parity, and the color of each number represents the parity bin (zero, low, or high).

Additional file 3: Supplemental Figure 3. Shannon alpha diversity increases throughout pregnancy across all parity groups. Shannon alpha diversity was calculated for each sample at each time point. Alpha diversity trends upwards over the course of pregnancy in all three parity groups. Parity does not associate with alpha diversity.

Additional file 4: Supplemental Figure 4. Weighted UniFrac beta diversity shows that parity affects the gut microbiota trajectory during gestation. A) Weighted UniFrac beta diversity was calculated between each sample and the Day 37 sample from the same individual. B) Weighted UniFrac beta diversity was calculated between each sample and the previous week's sample from the same individual.

Additional file 5: Supplemental Figure 5. The relative abundance of key bacterial taxa changes throughout gestation. A spline-fitting model was performed for the top five most important taxa from the DMM (Fig

1D) and the top five most important taxa from the maturity index (Fig 1B). The relative abundance of all ten taxa changed significantly during gestation $(P<0.01)$. Throughout gestation, the relative abundance of two taxa, the genus Phascolarctobacterium and the family Rikenellaceae, were significantly associated with parity $(P<0.05)$.

Additional file 6: Supplemental Figure 6. Sows and piglets have significantly different gut microbiome compositions. Shotgun metagenomic sequencing was performed for fecal samples from 18 mother sows that were collected at days 37 and 114 of gestation, and from fecal swabs from 3 offspring of each pig collected 10 days after delivery. A) An NMDS plot representing the Bray-Curtis beta diversity between the 90 samples (36 sow and 54 piglet) was generated. The microbiome compositions of sows are significantly different from that of piglets $(P=1 \mathrm{e}-5)$. B) A heatmap was generated using the 32 bacterial species with relative abundance $>1 \%$ among either sows or piglets. For each sample, the heatmap shows the relative abundance of each species. Consistent with the NMDS plot, the heatmap separates into two major phylogenetic branches, with sows on the left and piglets on the right. Four sow samples cluster within the piglet branch and are noted below the heatmap.

Additional file 7: Supplemental Figure 7. The relative abundance of bacterial taxa in a sow does not correlate with the relative abundance of that taxa in her offspring. Linear regression analyses was performed for 
each of the 8 bacterial species with average relative abundance $>1 \%$ across all samples and that were present in at least two-thirds of all samples. For each piglet, the relative abundance of each bacterial species in its mother at Day 114 was plotted (X-axis) against the relative abundance of that species in the piglet 10 days after delivery (Y-axis). Fecal swabs from three piglets from each sow were sequenced. There were no significant correlations between bacterial species in mother and offspring in any of the eight species (Adj. $P>0.3$ ).

Additional file 8: Supplemental Figure 8. There is no evidence that Escherichia coli in piglets were inherited from the maternal gut. A neighbor-joining phylogeny was reconstructed using 34 E. coli strains extracted from the shotgun metagenomic sequencing data from piglets and sows likely harboring only a single $E$. coli strain. Three sets of $E$. coli from different piglet-sow sets are highlighted in orange, cyan, and red. For example, highlighted in orange, the 3 piglets born to Sow 214 share similar E. coli genotypes, but immediately prior to birth (Day 114), the sow's gut harbored a very different E. coli genotype. Similarly, in cyan, Sow 4074 and her piglet harbor different E. coli genotypes. Finally, in red, three piglets of Sow 1962 harbor E. coli genotypes with a most recent common ancestor at the root of the phylogeny. E. coli was chosen because it is the microbe found in the highest abundance across both sows and piglets, thus providing the deepest coverage of strains.

Additional file 9: Supplemental Figure 9. The number of piglets per litter, the interpregnancy interval, and litter weight are not significantly different between parity groups. A) The number of piglets per litter across parity groups is constant. B) The number of days between weaning and insemination does not differ between parity groups. C) The combined weight of each litter is not significantly different between parity groups. All $P$-values are $>0.05$.

\section{Acknowledgements}

We thank Dr. Shuai Wang and Ms. Elise Krespan for providing feedback on data presentation and analyses, and we thank Dr. Christopher J. Stewart (Newcastle University) for advice on DMM analysis and code.

\section{Authors' contributions}

MKP, TDP, and DPB designed the study. MKP collected samples. MKP and TDP oversaw operations in the swine facility. ASFB, KO, YC, SM, and LR processed the samples. AMM, MCS, and ASFB prepared libraries and carried out sequencing. ASFB and DPB analyzed the data and wrote the paper. MKP, TDP, and RNB assisted with the writing and data interpretation. The authors read and approved the final manuscript.

\section{Funding}

A Tobacco Formula grant provided partial support for the project and for ASFB and RNB. This Tobacco Formula grant is under the Commonwealth Universal Research Enhancement (CURE) program with the grant number SAP \# 4100068710. The funders had no role in data collection and analysis, decision to publish, or preparation of the manuscript.

\section{Availability of data and materials}

All $16 \mathrm{~S}$ and shotgun metagenomic sequencing data are publicly available on the Sequence Read Archive (SRA) under the study accession numbers PRJNA644935 and PRJNA645191, respectively. All code and software used to process and analyze the data is available as a fully reproducible computing environment on Code Ocean (https://codeocean.com/capsule/8021926/tree).

\section{Declarations}

\section{Ethics approval and consent to participate}

Research was completed under permits issued by the Institutional Care and Use Committee of the University of Pennsylvania as IACUC Protocol \#806059 under The Guide for the Care and Use of Laboratory Animals.

\section{Consent for publication}

All authors of this work concur with this submission, and the data presented have not been previously reported nor are they under consideration for publication elsewhere.

\section{Competing interests}

The authors have no competing interests to declare.

\section{Author details}

${ }^{1}$ Department of Pathobiology, School of Veterinary Medicine, University of Pennsylvania, Philadelphia, PA, USA. ²Department of Pediatric Gastroenterology Hepatology and Nutrition, Children's Hospital of Philadelphia, Philadelphia, PA 19104, USA. ${ }^{3}$ Department of Clinical Studies New Bolton Center, School of Veterinary Medicine, University of Pennsylvania, Philadelphia, PA, USA.

Received: 11 December 2020 Accepted: 6 May 2021

Published online: 27 June 2021

\section{References}

1. Elovitz MA, Gajer P, Riis V, Brown AG, Humphrys MS, Holm JB, et al. Cervicovaginal microbiota and local immune response modulate the risk of spontaneous preterm delivery. Nat Commun. 2019;10(1):1305. https://doi. org/10.1038/s41467-019-09285-9

2. DiGiulio DB, Callahan BJ, McMurdie PJ, Costello EK, Lyell DJ, Robaczewska A, et al. Temporal and spatial variation of the human microbiota during pregnancy. Proc Natl Acad Sci USA. 2015;112(35):11060-5. https://doi.org/1 $0.1073 /$ pnas. 1502875112

3. Fettweis JM, Serrano MG, Brooks JP, Edwards DJ, Girerd PH, Parikh HI, et al. The vaginal microbiome and preterm birth. Nat Med. 2019;25(6):1012-21. https://doi.org/10.1038/s41591-019-0450-2.

4. Blencowe $H$, Cousens S, Chou D, Oestergaard M, Say L, Moller A-B, et al. Born too soon: the global epidemiology of 15 million preterm births. Reprod Health. 2013;10(Suppl 1):S2. https://doi.org/10.1186/1742-4755-10S1-S2.

5. Gomez de Agüero M, Ganal-Vonarburg SC, Fuhrer T, Rupp S, Uchimura Y, Li $\mathrm{H}$, et al. The maternal microbiota drives early postnatal innate immune development. Science. 2016;351:1296-302. https://doi.org/10.1126/ science.aad2571.

6. Kimura I, Miyamoto J, Ohue-Kitano R, Watanabe K, Yamada T, Onuki M, et al. Maternal gut microbiota in pregnancy influences offspring metabolic phenotype in mice. Science. 2020;367(6481):eaaw8429. https://doi.org/1 $0.1126 /$ science.aaw8429.

7. Rackaityte E, Halkias J, Fukui EM, Mendoza VF, Hayzelden C, Crawford ED, et al. Viable bacterial colonization is highly limited in the human intestine in utero. Nat Med. 2020;26(4):599-607. https://doi.org/10.1038/s41591-0200761-3.

8. Korpela K, Renko M, Paalanne N, Vänni P, Salo J, Tejesvi M, et al. Microbiome of the first stool after birth and infantile colic. Pediatr Res. 2020;88(5):776-83. https://doi.org/10.1038/s41390-020-0804-y.

9. Korpela K, Renko M, Vänni P, Paalanne N, Salo J, Tejesvi MV, et al. Microbiome of the first stool and overweight at age 3 years: a prospective cohort study. Pediatr Obes. 2020;15(11):e12680. https://doi.org/10.1111/ ijpo.12680.

10. Menon R. Spontaneous preterm birth, a clinical dilemma: etiologic, pathophysiologic and genetic heterogeneities and racial disparity. Acta Obstet Gynecol Scand. 2008;87(6):590-600. https://doi.org/10.1080/0001634 0802005126.

11. Reagan PB, Salsberry PJ. Race and ethnic differences in determinants of preterm birth in the USA: broadening the social context. Soc Sci Med. 2005; 60(10):2217-28. https://doi.org/10.1016/j.socscimed.2004.10.010.

12. Russell SL, Ickovics JR, Yaffee RA. Exploring potential pathways between parity and tooth loss among American women. Am J Public Health. 2008; 98(7):1263-70. https://doi.org/10.2105/AJPH.2007.124735.

13. Bogado Pascottini O, Spricigo JFW, Van Schyndel SJ, Mion B, Rousseau J, Weese JS, et al. Effects of parity, blood progesterone, and non-steroidal antiinflammatory treatment on the dynamics of the uterine microbiota of healthy postpartum dairy cows. PLoS One. 2021;16(2):e0233943. https://doi. org/10.1371/journal.pone.0233943.

14. Pitta DW, Indugu N, Kumar S, Vecchiarelli B, Sinha R, Baker LD, et al. Metagenomic assessment of the functional potential of the rumen microbiome in Holstein dairy cows. Anaerobe. 2016;38:50-60. https://doi. org/10.1016/j.anaerobe.2015.12.003.

15. Kumbhare SV, Patangia DV, Mongad DS, Bora A, Bavdekar AR, Shouche YS Gut microbial diversity during pregnancy and early infancy: an exploratory 
study in the Indian population. FEMS Microbiol Lett. 2020;367(3). https://doi. org/10.1093/femsle/fnaa022.

16. Koren O, Goodrich JK, Cullender TC, Spor A, Laitinen K, Bäckhed HK, et al. Host remodeling of the gut microbiome and metabolic changes during pregnancy. Cell. 2012;150(3):470-80. https://doi.org/10.1016/j.cell.2012.07. 008.

17. Rothschild D, Weissbrod O, Barkan E, Kurilshikov A, Korem T, Zeevi D, et al. Environment dominates over host genetics in shaping human gut microbiota. Nature. 2018;555(7695):210-5. https://doi.org/10.1038/nature2 5973.

18. Lundgren SN, Madan JC, Emond JA, Morrison HG, Christensen BC, Karagas $M R$, et al. Maternal diet during pregnancy is related with the infant stool microbiome in a delivery mode-dependent manner. Microbiome. 2018;6(1): 109. https://doi.org/10.1186/s40168-018-0490-8.

19. Wu GD, Chen J, Hoffmann C, Bittinger K, Chen Y-Y, Keilbaugh SA, et al. Linking long-term dietary patterns with gut microbial enterotypes. Science. 2011;334(6052):105-8. https://doi.org/10.1126/science.1208344.

20. Gurry T, HST Microbiome Consortium*, Gibbons SM, LTT N, Kearney SM, Ananthakrishnan A, et al. Predictability and persistence of prebiotic dietary supplementation in a healthy human cohort. Sci Rep. 2018;8(1):12699. https://doi.org/10.1038/s41598-018-30783-1.

21. Smits SA, Leach J, Sonnenburg ED, Gonzalez CG, Lichtman JS, Reid G, et al. Seasonal cycling in the gut microbiome of the Hadza hunter-gatherers of Tanzania. Science. 2017;357(6353):802-6. https://doi.org/10.1126/science.aa n4834.

22. Hansen MEB, Rubel MA, Bailey AG, Ranciaro A, Thompson SR, Campbell MC, et al. Population structure of human gut bacteria in a diverse cohort from rural Tanzania and Botswana. Genome Biol. 2019;20(1):16. https://doi.org/1 0.1186/s13059-018-1616-9.

23. Heinritz SN, Mosenthin R, Weiss E. Use of pigs as a potential model for research into dietary modulation of the human gut microbiota. Nutr Res Rev. 2013;26(2):191-209. https://doi.org/10.1017/50954422413000152.

24. Kim HB, Isaacson RE. The pig gut microbial diversity: understanding the pig gut microbial ecology through the next generation high throughput sequencing. Vet Microbiol. 2015;177(3-4):242-51. https://doi.org/10.1016/j. vetmic.2015.03.014.

25. Li Y, Guo Y, Wen Z, Jiang X, Ma X, Han X. Weaning stress perturbs gut microbiome and its metabolic profile in piglets. Sci Rep. 2018;8(1):18068 https://doi.org/10.1038/s41598-018-33649-8.

26. Liu H, Hou C, Li N, Zhang X, Zhang G, Yang F, et al. Microbial and metabolic alterations in gut microbiota of sows during pregnancy and lactation. FASEB J. 2019;33(3):4490-501. https://doi.org/10.1096/fi.201801221RR.

27. Frese SA, Parker K, Calvert CC, Mills DA. Diet shapes the gut microbiome of pigs during nursing and weaning. Microbiome. 2015;3(1):28. https://doi. org/10.1186/s40168-015-0091-8

28. Yan H, Potu R, Lu H, Vezzoni de Almeida V, Stewart T, Ragland D, et al. Dietary fat content and fiber type modulate hind gut microbial community and metabolic markers in the pig. PLoS One. 2013;8(4):e59581. https://doi. org/10.1371/journal.pone.0059581.

29. Wang X, Tsai T, Deng F, Wei X, Chai J, Knapp J, et al. Longitudinal investigation of the swine gut microbiome from birth to market reveals stage and growth performance associated bacteria. Microbiome. 2019;7(1): 109. https://doi.org/10.1186/s40168-019-0721-7.

30. Bergamaschi M, Tiezzi F, Howard J, Huang YJ, Gray KA, Schillebeeckx C, et al. Gut microbiome composition differences among breeds impact feed efficiency in swine. Microbiome. 2020;8(1):110. https://doi.org/10.1186/s401 68-020-00888-9.

31. Bokulich NA, Dillon MR, Bolyen E, Kaehler BD, Huttley GA, Caporaso JG. q2sample-classifier: machine-learning tools for microbiome classification and regression. J Open Res Softw. 2018;3. https://doi.org/10.21105/joss.00934.

32. Subramanian S, Huq S, Yatsunenko T, Haque R, Mahfuz M, Alam MA, et al. Persistent gut microbiota immaturity in malnourished Bangladeshi children. Nature. 2014;510(7505):417-21. https://doi.org/10.1038/nature13421.

33. Mitchell C, Hogstrom L, Bryant AM, Bergerat A, Cher A, Pochan S, et al. Delivery mode impacts newborn gut colonization efficiency. BioRxiv. 2020. https://doi.org/10.1101/2020.01.29.919993.

34. Yassour M, Jason E, Hogstrom $\sqcup$, Arthur TD, Tripathi $S$, Siljander $H$, et al. Strain-level analysis of mother-to-child bacterial transmission during the first few months of life. Cell Host Microbe. 2018;24:146-154.e4. https://doi.org/1 0.1016/..chom.2018.06.007.
35. Truong DT, Tett A, Pasolli E, Huttenhower C, Segata N. Microbial strain-level population structure and genetic diversity from metagenomes. Genome Res. 2017;27(4):626-38. https://doi.org/10.1101/gr.216242.116.

36. Gibbons SM, Duvallet C, Alm EJ. Correcting for batch effects in case-control microbiome studies. PLoS Comput Biol. 2018;14(4):e1006102. https://doi. org/10.1371/journal.pcbi.1006102.

37. Ho NT, Li F, Wang S, Kuhn L. metamicrobiomeR: an R package for analysis of microbiome relative abundance data using zero-inflated beta GAMLSS and meta-analysis across studies using random effects models. BMC Bioinformatics. 2019;20(1):188. https://doi.org/10.1186/s12859-019-2744-2.

38. Oliveira FS, Brestelli J, Cade S, Zheng J, lodice J, Fischer S, et al. MicrobiomeDB: a systems biology platform for integrating, mining and analyzing microbiome experiments. Nucleic Acids Res. 2018;46(D1):D684-91. https://doi.org/10.1093/nar/gkx1027.

39. Kim SW. Recent advances in sow nutrition. R Bras Zootec. 2010;39(suppl spe):303-10. https://doi.org/10.1590/S1516-35982010001300033.

40. Rey FE, Gonzalez MD, Cheng J, Wu M, Ahern PP, Gordon Jl. Metabolic niche of a prominent sulfate-reducing human gut bacterium. Proc Natl Acad Sci U S A. 2013;110(33):13582-7. https://doi.org/10.1073/pnas.1312524110.

41. Finegold SM, Song Y, Liu C, Hecht DW, Summanen P, Könönen E, et al. Clostridium clostridioforme: a mixture of three clinically important species. Eur J Clin Microbiol Infect Dis. 2005;24(5):319-24. https://doi.org/10.1007/s1 0096-005-1334-6.

42. Martín R, Heilig GHJ, Zoetendal EG, Smidt H, Rodríguez JM. Diversity of the Lactobacillus group in breast milk and vagina of healthy women and potential role in the colonization of the infant gut. J Appl Microbiol. 2007; 103(6):2638-44. https://doi.org/10.1111/j.1365-2672.2007.03497.x.

43. Jost T, Lacroix C, Braegger CP, Rochat F, Chassard C. Vertical motherneonate transfer of maternal gut bacteria via breastfeeding. Environ Microbiol. 2014;16(9):2891-904. https://doi.org/10.1111/1462-2920.12238.

44. Pannaraj PS, Li F, Cerini C, Bender JM, Yang S, Rollie A, et al. Association between breast milk bacterial communities and establishment and development of the infant gut microbiome. JAMA Pediatr. 2017;171(7):64754. https://doi.org/10.1001/jamapediatrics.2017.0378.

45. Xu J, Lawley B, Wong G, Otal A, Chen L, Ying TJ, et al. Ethnic diversity in infant gut microbiota is apparent before the introduction of complementary diets. Gut Microbes. 2020;11(5):1362-73. https://doi.org/10.1 080/19490976.2020.1756150.

46. Nyangahu DD, Darby M, Havyarimana E, Brown BP, Horsnell W, Jaspan HB. Preconception helminth infection alters offspring microbiota and immune subsets in a mouse model. Parasite Immunol. 2020;42(9):e12721. https://doi. org/10.1111/pim.12721.

47. Bachour P, Yafawi R, Jaber F, Choueiri E, Abdel-Razzak Z. Effects of smoking, mother's age, body mass index, and parity number on lipid, protein, and secretory immunoglobulin A concentrations of human milk. Breastfeed Med. 2012;7(3):179-88. https://doi.org/10.1089/bfm.2011.0038.

48. Lima SF, Teixeira AGV, Lima FS, Ganda EK, Higgins CH, Oikonomou G, et al. The bovine colostrum microbiome and its association with clinical mastitis. J Dairy Sci. 2017;100(4):3031-42. https://doi.org/10.3168/jds.2016-11604.

49. Quan J, Wu Z, Ye Y, Peng L, Wu J, Ruan D, et al. Metagenomic characterization of intestinal regions in pigs with contrasting feed efficiency. Front Microbiol. 2020;11:32. https://doi.org/10.3389/fmicb.2020.00032.

50. Wang A, Yu H, Gao X, Li X, Qiao S. Influence of Lactobacillus fermentum 15007 on the intestinal and systemic immune responses of healthy and $E$. coli challenged piglets. Antonie Van Leeuwenhoek. 2009;96(1):89-98. https://doi.org/10.1007/s10482-009-9339-2.

51. Gaggia F, Mattarelli P, Biavati B. Probiotics and prebiotics in animal feeding for safe food production. Int J Food Microbiol. 2010;141(Suppl 1):S15-28. https://doi.org/10.1016/.i.jfoodmicro.2010.02.031.

52. Bergamaschi M, Maltecca C, Schillebeeckx C, McNulty NP, Schwab C, Shull C, et al. Heritability and genome-wide association of swine gut microbiome features with growth and fatness parameters. Sci Rep. 2020;10(1):10134. https://doi.org/10.1038/s41598-020-66791-3.

53. Pluske JR. Feed-and feed additives-related aspects of gut health and development in weanling pigs. J Anim Sci Biotechnol. 2013;4(1):1. https:// doi.org/10.1186/2049-1891-4-1.

54. Urbaniak C, Cummins J, Brackstone M, Macklaim JM, Gloor GB, Baban CK, et al. Microbiota of human breast tissue. Appl Environ Microbiol. 2014 80(10):3007-14. https://doi.org/10.1128/AEM.00242-14.

55. Dao MC, Everard A, Aron-Wisnewsky J, Sokolovska N, Prifti E, Verger EO, et al. Akkermansia muciniphila and improved metabolic health during a 
dietary intervention in obesity: relationship with gut microbiome richness and ecology. Gut. 2016;65(3):426-36. https://doi.org/10.1136/gutjnl-2014-3 08778.

56. Cani PD, de Vos WM. Next-generation beneficial microbes: the case of Akkermansia muciniphila. Front Microbiol. 2017;8:1765. https://doi.org/10.33 89/fmicb.2017.01765

57. Yang H, Xiang Y, Robinson K, Wang J, Zhang G, Zhao J, et al. Gut microbiota is a major contributor to adiposity in pigs. Front Microbiol. 2018; 9:3045. https://doi.org/10.3389/fmicb.2018.03045.

58. Lord SJ, Bernstein L, Johnson KA, Malone KE, McDonald JA, Marchbanks PA, et al. Breast cancer risk and hormone receptor status in older women by parity, age of first birth, and breastfeeding: a case-control study. Cancer Epidemiol Biomarkers Prev. 2008;17(7):1723-30. https://doi.org/10.1158/1 055-9965.EPI-07-2824.

59. Islami F, Liu Y, Jemal A, Zhou J, Weiderpass E, Colditz G, et al. Breastfeeding and breast cancer risk by receptor status--a systematic review and metaanalysis. Ann Oncol. 2015;26(12):2398-407. https://doi.org/10.1093/annonc/ mdv379.

60. Kozuki N, Lee ACC, Silveira MF, Sania A, Vogel JP, Adair L, et al. The associations of parity and maternal age with small-for-gestational-age, preterm, and neonatal and infant mortality: a meta-analysis. BMC Public Health. 2013;13(Suppl 3):S2. https://doi.org/10.1186/1471-2458-13-S3-S2.

61. Tsilidis KK, Allen NE, Key TJ, Dossus L, Lukanova A, Bakken K, et al. Oral contraceptive use and reproductive factors and risk of ovarian cancer in the European Prospective Investigation into Cancer and Nutrition. Br J Cancer. 2011;105(9):1436-42. https://doi.org/10.1038/bjc.2011.371.

62. Bae JB, Lipnicki DM, Han JW, Sachdev PS, Kim TH, Kwak KP, et al. Does parity matter in women's risk of dementia? A COSMIC collaboration cohort study. BMC Med. 2020;18(1):210. https://doi.org/10.1186/s12916-020-01671-1.

63. Urzua U, Chacon C, Lizama L, Sarmiento S, Villalobos P, Kroxato B, et al. Parity history determines a systemic inflammatory response to spread of ovarian cancer in naturally aged mice. Aging Dis. 2017;8:546-57. https://doi. org/10.14336/AD.2017.0110.

64. Arslan AA, Zeleniuch-Jacquotte A, Lukanova A, Afanasyeva Y, Katz J, Levitz $M$, et al. Effects of parity on pregnancy hormonal profiles across ethnic groups with a diverse incidence of breast cancer. Cancer Epidemio Biomarkers Prev. 2006;15(11):2123-30. https://doi.org/10.1158/1055-9965.EPI06-0470.

65. Barrat F, Lesourd B, Boulouis HJ, Thibault D, Vincent-Naulleau S, Gjata B, et al. Sex and parity modulate cytokine production during murine ageing. Clin Exp Immunol. 1997;109(3):562-8. https://doi.org/10.1046/j.1365-2249.1 997.4851387.x.

66. Gaillard R, Rurangirwa AA, Williams MA, Hofman A, Mackenbach JP, Franco $\mathrm{OH}$, et al. Maternal parity, fetal and childhood growth, and cardiometabolic risk factors. Hypertension. 2014;64(2):266-74. https://doi.org/10.1161/ HYPERTENSIONAHA.114.03492.

67. National Research Council. Nutrient requirements of swine: eleventh revised edition. Washington, D.C: National Academies Press; 2012. https://doi.org/1 $0.17226 / 13298$.

68. Berry ASF, Johnson K, Martins R, Sullivan MC, Farias Amorim C, Putre A, et al Natural infection with giardia is associated with altered community structure of the human and canine gut microbiome. mSphere. 2020;5. https://doi.org/10.1128/mSphere.00670-20.

69. Kozich JJ, Westcott SL, Baxter NT, Highlander SK, Schloss PD. Development of a dual-index sequencing strategy and curation pipeline for analyzing amplicon sequence data on the MiSeq Illumina sequencing platform. Appl Environ Microbiol. 2013;79(17):5112-20. https://doi.org/10.1128/AEM.01 043-13.

70. Bolyen E, Rideout JR, Dillon MR, Bokulich NA, Abnet CC, Al-Ghalith GA, et al. Reproducible, interactive, scalable and extensible microbiome data science using QIIME 2. Nat Biotechnol. 2019;37(8):852-7. https://doi.org/10.1038/s41 587-019-0209-9.

71. Callahan BJ, McMurdie PJ, Rosen MJ, Han AW, Johnson AJA, Holmes SP. DADA2: high-resolution sample inference from Illumina amplicon data. Nat Methods. 2016;13(7):581-3. https://doi.org/10.1038/nmeth.3869.

72. Katoh K, Misawa K, Kuma K, Miyata T. MAFFT: a novel method for rapid multiple sequence alignment based on fast Fourier transform. Nucleic Acids Res. 2002;30(14):3059-66. https://doi.org/10.1093/nar/gkf436.

73. Price MN, Dehal PS, Arkin AP. FastTree 2 - approximately maximumlikelihood trees for large alignments. PLoS One. 2010;5(3):e9490. https://doi. org/10.1371/journal.pone.0009490.
74. Lozupone C, Knight R. UniFrac: a new phylogenetic method for comparing microbial communities. Appl Environ Microbiol. 2005;71(12):8228-35. https://doi.org/10.1128/AEM.71.12.8228-8235.2005.

75. Sorensen T. A method of establishing groups of equal amplitude in plant sociology based on similarity of species content. Kongelige Danske Videnskabernes Selskab; 1948.

76. Benjamini Y, Hochberg Y. Controlling the false discovery rate: a practical and powerful approach to multiple testing. J R Stat Soc Series B (Methodological). 1995;57(1):289-300. https://doi.org/10.1111/j.2517-6161.1 995.tb02031.x.

77. Bokulich NA, Kaehler BD, Rideout JR, Dillon M, Bolyen E, Knight R, et al. Optimizing taxonomic classification of marker-gene amplicon sequences with QIIME 2's q2-feature-classifier plugin. Microbiome. 2018;6(1):90. https:// doi.org/10.1186/s40168-018-0470-z.

78. Quast C, Pruesse E, Yilmaz P, Gerken J, Schweer T, Yarza P, et al. The SILVA ribosomal RNA gene database project: improved data processing and webbased tools. Nucleic Acids Res. 2013;41(Database issue):D590-6. https://doi. org/10.1093/nar/gks1219

79. Yilmaz P, Parfrey LW, Yarza P, Gerken J, Pruesse E, Quast C, et al. The SILVA and "All-species Living Tree Project (LTP)" taxonomic frameworks. Nucleic Acids Res. 2014;42(Database issue):D643-8. https://doi.org/10.1093/nar/gkt12 09.

80. Bokulich NA, Dillon MR, Zhang Y, Rideout JR, Bolyen E, Li H, et al. q2longitudinal: longitudinal and paired-sample analyses of microbiome data. mSystems. 2018;3. https://doi.org/10.1128/mSystems.00219-18.

81. Zhang Y, Han SW, Cox LM, Li H. A multivariate distance-based analytic framework for microbial interdependence association test in longitudinal study. Genet Epidemiol. 2017;41(8):769-78. https://doi.org/10.1002/gepi.22 065.

82. Holmes I, Harris K, Quince C. Dirichlet multinomial mixtures: generative models for microbial metagenomics. PLoS One. 2012;7(2):e30126. https:// doi.org/10.1371/journal.pone.0030126.

83. Stewart CJ, Ajami NJ, O'Brien JL, Hutchinson DS, Smith DP, Wong MC, et al. Temporal development of the gut microbiome in early childhood from the TEDDY study. Nature. 2018;562(7728):583-8. https://doi.org/10.1038/s41586018-0617-x.

84. Shields-Cutler RR, Al-Ghalith GA, Yassour M, Knights D. Splinectomer enables group comparisons in longitudinal microbiome studies. Front Microbiol. 2018;9:785. https://doi.org/10.3389/fmicb.2018.00785.

85. Bolger AM, Lohse M, Usadel B. Trimmomatic: a flexible trimmer for Illumina sequence data. Bioinformatics. 2014;30(15):2114-20. https://doi.org/10.1093/ bioinformatics/btu170.

86. Segata N, Waldron L, Ballarini A, Narasimhan V, Jousson O, Huttenhower C Metagenomic microbial community profiling using unique clade-specific marker genes. Nat Methods. 2012;9(8):811-4. https://doi.org/10.1038/ nmeth.2066.

87. Oksanen J, Blanchet FG, Friendly M, Kindt R, Legendre P, McGlinn D, et al. vegan: Community Ecology Package. Computer software. R; 2019.

88. R Core Team. R: A language and environment for statistical computing. Computer software. Vienna: R Foundation for Statistical Computing; 2018.

89. Segata N, Izard J, Waldron L, Gevers D, Miropolsky L, Garrett WS, et al. Metagenomic biomarker discovery and explanation. Genome Biol. 2011; 12(6):R60. https://doi.org/10.1186/gb-2011-12-6-r60

90. Wickham H. ggplot2: elegant graphics for data analysis. New York: Springer, c2009; 2009.

91. Pedersen TL. patchwork: The Composer of ggplots. Computer software. R; 2017.

92. Arnold JB. ggthemes: Extra Themes, Scales and Geoms for "ggplot2." Computer software. R; 2019.

93. Kumar S, Stecher G, Tamura K. MEGA7: molecular evolutionary genetics analysis version 7.0 for bigger datasets. Mol Biol Evol. 2016;33(7):1870-4. https://doi.org/10.1093/molbev/msw054.

\section{Publisher's Note}

Springer Nature remains neutral with regard to jurisdictional claims in published maps and institutional affiliations. 